\title{
On the geometry of the string landscape and the swampland
}

\section{Citation}

Ooguri, Hirosi, and Cumrun Vafa. 2007. "On the Geometry of the String Landscape and the Swampland." Nuclear Physics B 766 (1-3): 21-33. https://doi.org/10.1016/ j.nuclphysb.2006.10.033.

\section{Permanent link}

http://nrs.harvard.edu/urn-3:HUL.InstRepos:41385093

\section{Terms of Use}

This article was downloaded from Harvard University's DASH repository, and is made available under the terms and conditions applicable to Other Posted Material, as set forth at http:// nrs.harvard.edu/urn-3:HUL.InstRepos:dash.current.terms-of-use\#LAA

\section{Share Your Story}

The Harvard community has made this article openly available.

Please share how this access benefits you. Submit a story.

Accessibility 
CALT-68-2600, HUTP-06/A017

\title{
On the Geometry of the String Landscape and the Swampland
}

\author{
Hirosi Ooguri ${ }^{1}$ and Cumrun Vafa ${ }^{2}$ \\ ${ }^{1}$ California Institute of Technology \\ Pasadena, CA 91125, USA \\ ${ }^{2}$ Jefferson Physical Laboratory, Harvard University \\ Cambridge, MA 02138, USA
}

\begin{abstract}
We make a number of conjectures about the geometry of continuous moduli parameterizing the string landscape. In particular we conjecture that such moduli are always given by expectation value of scalar fields and that moduli spaces with finite non-zero diameter belong to the swampland. We also conjecture that points at infinity in a moduli space correspond to points where an infinite tower of massless states appear, and that near these regions the moduli space is negatively curved. We also propose that there is no non-trivial 1-cycle of minimum length in the moduli space. This leads in particular to the prediction of the existence of a radially massive partner to the axion. These conjectures put strong constraints on inflaton potentials that can appear in a consistent quantum theory of gravity. Our conjectures are supported by a number of highly non-trivial examples from string theory. Moreover it is shown that these conditions can be violated if gravity is decoupled.
\end{abstract}

May 2006 


\section{Introduction}

The fact that string theory seems to offer a diverse range of possibilities for vacua has been viewed as a drawback for the theory: We cannot converge on a precise prediction for the theory. However despite this diversity of options for the string landscape, it has been pointed out in [1] that there are also a number of patterns that seem to emerge. Not every effective field theory that appears consistent seems to arise in string theory. It is natural to conjecture that these theories are not fully consistent as quantum gravitational theories. Such theories belong to the swampland. The condition to be on the swampland can be lifted if we decouple gravity. In other words a fully consistent quantum field theory cannot always be coupled to gravity. It is natural to conjecture that points on the swampland are "anomalous quantum gravitational theories," which are anomalous in a more subtle way, than has been discovered in the context of quantum field theories. It would be important to improve the restrictions for theories to arise in string theory. By tabulating such restrictions and finding the criteria that distinguish the swampland from the landscape one can hope to have a deeper understanding into the universality class of quantum gravitational theories. The main aim of this paper is to take some modest steps in this direction. For some related conjectures distinguishing swampland from the landscape see [2, 3, 田, 5].

One conjectural criterion to be on the string landscape is that the volume of the moduli space seems typically finite. There are, however, well known counter-examples to this seemingly general phenomenon. Consider compactification on a circle of radius $r$. The moduli space for the circle has a metric

$$
d s^{2}=\left(\frac{d r}{r}\right)^{2},
$$

and the volume integral is divergent. In [1], it was pointed out that this volume divergence correlates with the cutoff in mass. Namely, let $\epsilon$ be a fixed scale for the low energy effective theory and that we insist that all the higher massive scales have mass greater than $\epsilon$. For large $r$ and small $\epsilon$, the region of the moduli of the moduli space satisfying this constraint is specified by $r<1 / \epsilon$. Clearly the volume of this region of the moduli space is finite,

$$
\int^{1 / \epsilon} \frac{d r}{r}=-\log \epsilon .
$$

(The lower bound for the $r$ integral should also be regularized in a similar fashion, as will be clear in our examples.) We find the logarithmic volume divergence as we take the limit 
$\epsilon \rightarrow 0$. Thus, in this case, the volume divergence is related to the emergence of infinitely many extra light particles.

Even though this divergence might seem special to 1-dimensional moduli spaces, here we wish to formulate conjectures applicable to every moduli space $\mathcal{M}$ encountered in string theory. Our conjectures apply both to the moduli space of scalars, as well as the subspaces parameterizing minimum loci for the potentials defined on such spaces. In this way our conjectures can be viewed as very powerful constraints on what potentials can appear in a consistent theory of quantum gravity.

Our conjectures suggest the following picture for the moduli space of a consistent quantum gravitational theory: The moduli space is parameterized by expectation value of scalar fields. We conjecture that there are points infinitely far away from one another on the moduli space and that the points near infinity are points where a tower of massless modes appear (with exponentially small mass as a function of the distance to such points). Infinite distance singularities combined with finite volume typically imply negative curvatures. Thus, we conjecture that the curvature becomes negative near points at infinity. We also

propose that there are no non-trivial loops of minimum length in the moduli space of scalars. As we will elaborate later this is in line with the intuition that the duality groups are generated by discrete gauge symmetries realized at different points on the moduli space.

The organization of the paper is as follows: In section 2 we present the precise form for our conjectures. In section 3 we present examples of how string theory supports our conjectures and how if we decouple gravity we can violate them. In section 4 we discuss some field theoretic considerations related to our conjectures. In section 5 we end with some concluding thoughts.

\section{The Conjectures}

In this section we present our conjectures. We claim that our conjectures apply to consistent quantum theories of gravity with finite Planck mass in 4 and higher spacetime dimensions. We do not consider 3 or lower dimensions as gravity does not contain propagating degrees of freedom in these dimensions, though some of our conjectures may be applicable to 3 dimensional cases as well. Our conjectures should apply to string theories compactified to 4 and higher dimensions. But they should also apply to other consistent quantum gravity theories in these dimensions if they exist. 
Let $\mathcal{M}$ denote the moduli space of a consistent quantum gravity. Choosing a point $p \in \mathcal{M}$ corresponds to fixing the low energy effective Lagrangian for the theory. Different points in $\mathcal{M}$ are in different super-selection sectors. However, we could ask if we can vary $p$ locally in spacetime while keeping the asymptotic boundary conditions fixed at $p$. Our conjecture states that this is possible. In addition, if we consider a finite volume compactification down to 0 or 1 spatial dimensions (say on a torus), then we can vary the point $p$ and study $\mathcal{M}$. Our first conjecture states that $\mathcal{M}$ is given by the expectation value of scalar fields.

Conjecture 0: $\mathcal{M}$ is parameterized by inequivalent expectation values of massless scalar fields.

This conjecture in the context of string theory is so well known that one does not even bother to state it: There are no coupling constants in string theory; every parameter can be varied by changing the expectation value of a field. This did not have to be the case and one could a priori imagine a consistent quantum gravitational theory for which the parameters are fixed (and in particular cannot be varied locally). We conjecture that such gravitational theories cannot exist as a fully consistent quantum gravitational theory and belong to the swampland.

Given that points on $\mathcal{M}$ are given by the expectation value of scalar fields, we can use their kinetic term to define a metric on $\mathcal{M}$. We can then state our next conjecture dealing with the geometry of $\mathcal{M}$ : Let us assume that the dimension of $\mathcal{M}$ is not zero. Let us define $d\left(p_{1}, p_{2}\right)$ for two points $p_{1}, p_{2} \in \mathcal{M}$ as the distance of the shortest geodesic between them measured in Planck units. We conjecture that the distance can take an arbitrary large value, namely:

Conjecture 1: Choose any point $p_{0} \in \mathcal{M}$. For any positive $T$, there is another point $p \in \mathcal{M}$ such that $d\left(p, p_{0}\right)>T$.

More strongly, we claim that the limit $d\left(p, p_{0}\right) \rightarrow \infty$ is correlated with a breakdown of the low energy effective theory in a particular way:

Conjecture 2: Compared to the theory at $p_{0} \in \mathcal{M}$, the theory at $p$ with $d\left(p, p_{0}\right)>T$ has an infinite tower of light particles starting with mass of the order of $e^{-\alpha T}$ for some $\alpha>0$. In the $T \rightarrow \infty$ limit, the number of extra light particles of mass less than a fixed mass scale becomes infinite. 
In the first part of the conjecture 2, we are claiming that the low energy effective theory defined on a particular point on the moduli space makes sense only in a domain in a finite diameter from the point. In section 4 we will give a physical motivation for this part of the conjecture. If the breakdown of the low energy theory is associated with an infinite number of extra light particles, one-loop graphs of such particles can give rise to the distance divergence. If only a finite number of particles becomes light, they alone do not generate the distance divergence for dimensions higher than 2. This motivates the second part of the conjecture 2. Note that this conjecture in particular places strong constraints for inflationary models: We cannot have a slow roll inflation where the distance in the scalar moduli space is much bigger than Planck length and still use the same effective field theory.

Conjecture 3: The scalar curvature near the points at infinity is non-positive. (It is strictly negative if the dimension of the moduli space is greater than 1.)

The fact that the curvature is negative near infinity is correlated with the fact that there are points infinitely far away, even though the volume is finite.

One could consider a stronger conjecture claiming various sectional curvatures being negative near infinity. The geodesics deviation equation shows that neighboring geodesics tend to diverge when curvatures are negative. In particular, in a compact space, this leads to mixing of geodesics. More precisely, it is known that the geodesics flow in a compact space with negative sectional curvatures is ergodic [6]. Our conjectures here go in that direction. However they are not quite the same condition, as for instance, there are points on $\mathcal{M}$ arising in string theory where the sectional curvature is positive, as will be noted in the next section. Our conjecture here only refers to the scalar curvature being negative near infinity, which is weaker. Nevertheless, it could be true that the geodesic motion on the moduli space is ergodic. This could be potentially true, independently of the above conjecture, and would be worth investigating.

Conjecture 4: There is no non-trivial 1-cycle with minimum length within a given homotopy class in $\mathcal{M}$.

Suppose $\mathcal{M}$ is obtained from dividing a contractible Teichmüller $\mathcal{T}$ space by a group action $\Gamma$, as is often the case in string theory:

$$
\mathcal{M}=\mathcal{T} / \Gamma
$$


Then the duality group associated to $\mathcal{M}$ is $\Gamma$. Fixed points of $\Gamma$ have a nice physical significance: If $g \in \Gamma$ fixes a point $p \in \mathcal{T}$, then $g$ is a gauge symmetry at $p$. It seems to be the case that $\Gamma$ 's that appear in string theory are generated by group elements which have fixed points. In other words the entire $\Gamma$ can be viewed as a gauge symmetry which is broken as we move around in $\mathcal{M}$, and we recover different parts of it at different points of $\mathcal{M}$. If $\Gamma$ is indeed generated by elements with fixed points, then $\pi_{1}(\mathcal{M})$ would be trivial, as each loop which can be identified with an element $h \in \Gamma$ can be written as $h=\prod g_{i}$ and each $g_{i}$ has fixed points and so the corresponding segment of the path can be contracted to a point. So the entire path would be contractible, thus making $\mathcal{M}$ simply connected. Clearly in this case there is no 1-cycle of minimum length.

Another motivation for this conjecture is the following. Suppose we compactify the theory further down to $(1+1)$ dimensions and choose the space to be a circle. If there is a non-trivial 1-cycle in $\mathcal{M}$ with minimum length, we can use it to define a state with a non-zero global charge by going over the corresponding path on the circle. Since there are no global charges in a gravitational theory (to be consistent with no-hair theorems for black holes), this cannot happen. This suggests that at least the center of $\pi_{1}$ is trivial.

Conjecture 4 suggests there is a partner to the axion. The axion is massless before

we take into account instanton effects, and its has a compact moduli space of $S^{1}$. If we can separate the scale of the instanton effects from those at the Planck scale so that the instanton effects are taken into account in the low energy effective theory, the conjecture 4 would require that there exists another direction where $S^{1}$ shrinks to a point, i.e. a radial partner to the axion.

\section{Evidences for the Conjectures}

In the following, we will present evidences for these conjectures. We will also present examples in string theory that, if we decouple gravity by taking the internal volume to be infinitely large, then these constraints disappears. So it is only a constraint in the context of quantum theories of gravity. 


\subsection{Gravity Examples}

Example i) M-theory Compactified on $S^{1}$

In the 10-dimensional theory, which is equivalent to type IIA string, we have a massless scalar $r$ whose vev gives the radius of the circle. Since the metric on this space is $(d r / r)^{2}$, the geodesic distance between $r$ and $r_{0}$ is given by

$$
T=\left|\log \left(r / r_{0}\right)\right| .
$$

Since $r$ and $r_{0}$ can take arbitrary positive values, the conjecture 1 is clearly satisfied.

If we fix $r_{0}$ and take $T \rightarrow \infty$, we have either $r \rightarrow \infty$ or 0 . On the one hand, in the limit of $r \rightarrow \infty$, the M theory develops light Kaluza-Klein modes with mass $1 / r$ in the 11-dimensional frame. In the Einstein frame in 10 dimensions, which involves rescaling the metric by $r^{1 / 4}$, we find the lowest mass scale $\epsilon(T)$ is

$$
\epsilon(T) \sim r^{-1 / 8} r^{-1} \sim \exp \left(-\frac{9}{8} T\right)
$$

On the other hand, for the compactification at $r=r_{0} e^{-T}$, the radius $r \rightarrow 0$ and the theory again develop light states, namely type IIA strings, corresponding to membranes wrapping $S^{1}$. The tension of the string will scale as $r$, which means that their mass in 11-dimensional frame scales as $r^{1 / 2}$. Or in 10-dimensional Einstein frame as

$$
\epsilon(T) \sim r^{-1 / 8} r^{1 / 2}=r^{3 / 8} \sim \exp \left(-\frac{3}{8} T\right)
$$

So we see that this result is consistent with the conjecture 2 since in both cases infinitely many light modes appear as $T \rightarrow \infty$. Note that as this example demonstrates the coefficient of the exponent is not universal ( $9 / 8$ and $3 / 8$ in this case) even for a given dimension.

Example ii) Type IIB Strings in 10 Dimensions

The dilaton-axion moduli space is $S L(2, \mathbf{Z}) \backslash S L(2, \mathbf{R}) / S O(2)$, parametrized by $\tau=$ $\tau_{1}+i \tau_{2}$, where $\tau_{1}$ and $\tau_{2}$ are related to vev's of the axion and the dilaton. The metric in this space is

$$
d s^{2}=\frac{d \tau d \bar{\tau}}{(\operatorname{Im} \tau)^{2}} .
$$

This is a good example to illustrate the point of our conjectures since the volume of this moduli space is finite yet a geodesic length toward $i \infty$ is logarithmically divergent. As 
we take $\tau \rightarrow i \infty$ keeping $\tau_{0}$ finite, the geodesic length between $\tau$ and $\tau_{0}$ is approximately given by

$$
T \sim \log \left(\operatorname{Im} \tau / \operatorname{Im} \tau_{0}\right)
$$

Since light stringy excitations of mass $\sim e^{-\frac{1}{4} T}$ appear at $\tau$, this exemplifies the conjectures 1 and 2.

The metric (3.1) has a constant negative curvature, consistently with the conjecture 3. The curvature integral as well as the volume integral is finite, thanks to the quotienting by the S-duality symmetry $S L(2, \mathbf{Z})$. It is known that the geodesic flow on compact spaces with constant negative curvatures is ergodic [7]. In particular, if we consider a region $\tau_{2}>1 / \epsilon$ in the fundamental domain, generic geodesics will pass through it within finite geodesic time.

This moduli space is simply connected since $S L(2, \mathbf{Z})$ is generated by $S$ and $S T$ transformations $(S: \tau \rightarrow-1 / \tau, T: \tau \rightarrow \tau+1)$, both of which have fixed points in the fundamental domain, at $\tau=i$ and $\tau=\exp (2 \pi i / 3)$ respectively.

\section{Example iii) Type IIB Strings Compactified on $S^{1}$}

In this case the moduli space of compactification is characterized by the radius $r$ of $S^{1}$, in addition to the $\tau$ space discussed above. This example is similar to the example i, and we have

$$
T=\left|\log \left(r / r_{0}\right)\right|
$$

in string frame in 10 dimensions. We get light Kaluza-Klein modes for $r \rightarrow \infty$ and light winding modes for $r \rightarrow 0$. In the 9-dimensional Einstein frame, this translates to the existence of light mass scales for large $T$ given by

$$
\epsilon(T) \sim r^{-1 / 7} \exp (-T) \sim \exp \left(-\frac{8}{7} T\right) \quad \text { or } \quad \exp \left(-\frac{6}{7} T\right)
$$

Thus, both conjectures 1 and 2 are satisfied.

Example iv) Theories with 16 Supercharges

The classical moduli space of $K 3$ is

$$
\mathcal{M}_{K 3}^{(\text {classical })}=S O(3,19 ; \mathbf{Z}) \backslash S O(3,19) /(S O(2) \times S O(19)) .
$$


This is the relevant moduli space for the 7 dimensional theory obtained by compactifying M-theory on $K 3$. The physical moduli space of type II string on $K 3$ includes configurations of the NS-NS 2-form, the dilaton, and the R-R forms. In type IIA theory, the resulting moduli space, in addition to the coupling constant (as discussed in the example iii in the above), is

$$
\mathcal{K}_{K 3}^{I I A}=S O(4,20 ; \mathbf{Z}) \backslash S O(4,20) /(S O(4) \times S O(20))
$$

In type IIB theory, the coupling constant multiplet and other fields combine to give

$$
\mathcal{M}_{K_{3}}^{I I B}=S O(5,21 ; \mathbf{Z}) \backslash S O(5,21) /(S O(5) \times S O(21)) .
$$

In each of these moduli spaces, any point which is infinite distance away from points in the middle of the moduli space is dual to a decompactification limit. Therefore an infinite tower of light Kaluza-Klein modes appears at each of these points, in accord with the conjectures 1 and 2 .

These spaces have negative curvatures, satisfying the conjecture 3 . In general, if $\Gamma$ is a lattice in a connected semi-simple Lie group $G$ with finite center and $K$ is a maximal compact subgroup of $G$, generic geodesics are dense in the coset space $\Gamma \backslash G / K$ [8] leading to ergodicity.

For the $K 3$ moduli space, the discrete group $\Gamma$ is known to be generated by symmetry of the worldsheet CFT's [9, 10], and this supports the expectation from the conjecture 4 that these moduli spaces are simply connected.

The same is true for heterotic/type I string compactified on $T^{d}$, whose moduli space (in addition to the coupling constant) is

$$
S O(d, 16+d ; \mathbf{Z}) \backslash S O(d, 16+d ; \mathbf{R}) /(S O(d) \times S O(16+d)) .
$$

Example v) Compactifications of Type II Strings on Calabi-Yau threefolds

The moduli space splits into the vector multiplet moduli space, which has special Kähler structure, and the hypermultiplet moduli space, which is quaternionic Kähler. In the type IIB theory, the vector multiplet moduli space is parametrized complex structure of the Calabi-Yau manifold; in the type IIA theory, it is parametrized by complexified Kähler structure. The Kähler moduli space has at least one infinite distance singularity corresponding to the decompactification limit, where infinitely many Kaluza-Klein modes 
become light. By the mirror symmetry, we expect that the complex moduli space has an infinite distance singularity. This demonstrates the conjecture 1.

The curvature of the vector multiplet moduli space is not necessarily negative. However, one can see from explicit examples as that in [11] that the scalar curvature asymptotes to negative toward infinite distance singularities. In fact, this is a general fact. By using results in [12], one can estimate the behavior of the metric near singularities. Since the moduli space is orientable, the Einstein action can be written as

$$
\int_{\mathcal{M}} \sqrt{g} R \sim \int c_{1} \wedge k^{n-1},
$$

where $c_{1}$ is the first Chern class, $k$ is the Kähler form, and $n$ is the complex dimension of $\mathcal{M}$. This can be expressed as an integral of the same quantity of a compactification $\overline{\mathcal{M}}$ of $\mathcal{M}$, which is topological, plus contributions from singularities. One can show that infinite distance singularities always give strictly negative contributions to the integral, as expected from the conjecture 2. For Calabi-Yau manifolds with one Kähler moduli, the large size limit is always given by constant negative curvature (as the prepotential is dominated by $X_{1}^{3} / X_{0}$ in the standard notation).

Are Calabi-Yau moduli spaces simply connected? One way to test this is to see whether monodromies of period integrals are generated by transformations with fixed points represented by Calabi-Yau manifolds with symmetries. One subtlety is that such points can be at infinite distance away from the middle of the moduli space, in which case one has to be careful about how to define $\pi_{1}$. Indeed there is an examples of where the moduli space is complex one-dimensional with two different large complex structure limits 13.2. If we remove the two points in the limits, the moduli space has the topology of $\mathbf{C}^{*}$ and is not simply connected. In this case, the length of a circle that goes around the non-trivial $\pi_{1}$ of $\mathbf{C}^{*}$ vanishes as the circle approaches to either one of the large complex structure limits. This follows from the fact that the limits are infinite distances away yet the volume of the moduli space is finite. Therefore the conjecture 4 is still valid in this case.

As for the hypermulitplet moduli space, though a quaternionic Kähler manifold in general can have curvatures of any sign [14], it is known that those realized for hypermultiplet fields coupled to supergravity must have a negative scalar curvatures [15], satisfying

1 We would like to thank $\mathrm{Z}$. Lu for helping us make this estimate.

2 We thank D. Morrison for bringing this example into our attention. 
the conjecture 2. Note that the negativity of the scalar curvature in this case is a direct consequence of the local $\mathcal{N}=2$ supersymmetry, and therefore it applies to the hypermultiplet moduli space after perturbative and non-perturbative quantum corrections are taken into account as far as we consider the low energy effective theory with minimum number of derivatives.

\section{Example v) Compactifications to Four Dimensions with $\mathcal{N}=1$ Supersymmetry}

Little is known about the metric on $\mathcal{M}$ in this case. To our knowledge, the conjectures 1, 2, and 4 are consistent with all known examples. (Without information on the metric, it is difficult to test the conjecture 3 about the curvature of $\mathcal{M}$.) Consider for example the flux compactifications in [16]. In this construction, the classical superpotential generated by RR and NS-NS fluxes fixes all of the Kähler moduli and the complex structure moduli. There is one axion for each complex structure moduli multiplet that cannot be stabilized by the fluxes. In some models, these axions remain massless to all orders in $\alpha^{\prime}$ and $g_{s}$ expansions. However, in all known examples of this type, there are always instantons of the appropriate charges to lift all the remaining axions. ${ }^{3}$ Thus, it appears that there are obstructions in constructing models with compact moduli spaces with finite diameter (i.e. with a upper bound on $\left.d\left(p, p_{0}\right)\right)$ or with non-trivial fundamental group, and we regard this difficulty as in support of the conjectures 1,2 , and 4 .

\subsection{Non-Gravitational Counter-Examples}

We claim that the conjectures hold for theories with gravity with finite Planck scale. It is not difficult to construct non-gravitational field theory models which violate the conjectures. In this sense our conjectures focus on what aspects of a consistent quantum theory are required purely from requiring having gravity. For example, if the theory has a compact global symmetry $G$, as is typically the case in quantum field theories, and if it is spontaneously broken to a subgroup $H$, the moduli space $G / H$ has finite radius and the conjecture 1 fails in this case. Such spaces can have non-trivial fundamental groups, violating the conjecture 4 also. Since string theory cannot have any continuous global symmetry, such an example can exist only in the limit where we decouple gravity. In the following, we will present examples to show how the violation of the conjectures is explicitly tied to the decoupling of gravity.

3 We thank S. Kachru for discussion on this point. 
Examples a) Field Theories Living on Flat D-Branes in $R^{10}$.

The gauge theory on flat parallel $\mathrm{D} p$ branes in $R^{10}$ contains $(9-p)$ massless scalar fields in the adjoint representation of the gauge group. If we go to a generic direction away from the origin of the moduli space, we can go to infinity along geodesic without encountering extra massless particles. Therefore, this gives an example where the distance $d\left(p, p_{0}\right)$ can become infinite (and therefore the conjecture 1 holds), but no new massless particles appear there (and the conjecture 2 fails). In this case, the gravity degrees of freedom in the bulk 10 dimensions is decoupled. To have a finite Planck scale in $(p+1)$ dimensions along the branes, we need to compactify the transverse direction appropriately. This will change the structure of the gauge theory moduli space.

\section{Example b) Rigid Limit of $\mathcal{N}=2$ Theories in Four Dimensions}

We saw in the example $\mathrm{v}$ in section 3.1 that, in theories with local $\mathcal{N}=2$ supsersymmetries, the sign of curvature of vector multiplet moduli may be indefinite, but the scalar curvature becomes strictly negative near infinite distance singularities.

On the other hand, it is straightforward to see that, in non-gravitational theories with global $\mathcal{N}=2$ supersymmetry, the Ricci curvature of the vector multiplet moduli space is positive definite. In this case, the metric can be written as

$$
g_{i \bar{j}}=\operatorname{Im} \tau_{i j}, \quad \tau_{i j}=\partial_{i} \partial_{j} F(z),
$$

where $F(z)$ is the pre-potential. The Ricci curvature then is

$$
\begin{aligned}
R_{i \bar{j}} & =-\partial_{i} \bar{\partial}_{\bar{j}} \log \operatorname{det} g \\
& =\frac{1}{4} g^{k \bar{l}} g^{m \bar{n}}\left(\partial_{i} \tau_{k m}\right)\left(\bar{\partial}_{\bar{j}} \bar{\tau}_{\bar{l}}\right),
\end{aligned}
$$

which is manifestly positive definite. The essential step is to use the fact that $g_{i \bar{j}}$ is the imaginary part of $\tau_{i j}$, which is holomorphic in $z$.

It is instructive to see how the decoupling of the gravity leads to the positive definite Ricci curvature. In a theory with local $\mathcal{N}=2$ supersymmetry, the Kähler potential $K$ for the moduli space metric is given by [17]

$$
K=-\log \left(4 \mathcal{F}-4 \overline{\mathcal{F}}+\bar{z}^{\bar{i}} \partial_{i} \mathcal{F}-z^{i} \bar{\partial}_{\bar{i}} \overline{\mathcal{F}}\right),
$$


where $\mathcal{F}(z)$ is the pre-potential of the gravitational theory. The rigid limit can be taken by setting

$$
\mathcal{F}(z)=\frac{i}{8} M_{P l}^{2}+F(z)
$$

and let $M_{P l} \rightarrow \infty$. The Kähler potential then becomes, modulo terms that are purely holomorphic or anti-holomorphic,

$$
K=-\log i M_{P l}^{2}+\frac{1}{i M_{P l}^{2}}\left(\bar{z}^{\bar{i}} \partial_{i} F(z)-z^{i} \bar{\partial}_{\bar{i}} \bar{F}(\bar{z})\right)+\cdots
$$

in this limit, the metric $g_{i \bar{j}}$ becomes an imaginary part of the holomorphic $\tau_{i j}$ and the Ricci curvature is positive definite. This shows how the conjecture 2 can be violated by taking the decoupling limit $M_{P l} \rightarrow \infty$.

Example c) Type II Strings on Non-Compact Calabi-Yau Manifolds with wrapped branes

Another way to obtain field theories decoupled from gravity is to consider D-branes on cycles in a non-compact Calabi-Yau manifold $M$ and to take the low energy limit. For example, consider a brane at a point in $M$. Though the moduli space metric is not exactly equal to that of $M$ [18, 19], the metric computed using the gauge theory method of [20] is typically Ricci flat [21]. In particular, its scalar curvature does not necessarily become negative as we go infinite distance away from the origin of $M$.

As another example, consider a non-compact local model of Calabi-Yau manifold with small $\mathbf{P}^{2}$. The moduli space of a brane wrapping a 2-cycle in $\mathbf{P}^{2}$ is a copy of $\mathbf{P}^{2}$ (see 22] for a study of 2 branes in this geometry). Note that this space is compact (violating conjecture 1) and positively curved (violating conjecture 3). Note that cycles can shrink in a Calabi-Yau manifold only when they are positively curved. This may be related to the fact that scalar moduli coupled to gravity tend to be negatively curved, as suggested in the conjecture 3 .

Example d) D-brane Wrapping $T^{2}$

Consider type IIB compactified on $T^{2}$ to 8 dimensions. Consider a D5 brane wrapping $T^{2}$ and filling a $3+1$ dimensional flat subspace of 8 dimensions. The theory living on this 4 dimensions is a deformed $\mathcal{N}=4$ Yang-Mills, where out of the 6 scalars, two of them (corresponding to the Wilson lines on $T^{2}$ ) are periodic. The theory on this brane does not have a dynamical gravity in 4 dimensions (as the transverse dimensions are non-compact) and we have the moduli space of the theory given by

$$
R^{4} \times T^{2}
$$

This clearly violates conjecture 4 which states that the moduli space should have no nontrivial loops. $T^{2}$ has non-trivial loops. 


\section{Motivation from Effective Field Theories}

We would like to point out that there are phenomena in a low energy effective theory coupled to gravity that are closely related the property of moduli spaces discussed in the above.

\subsection{Infinite Distances and Appearance of a Tower of Light States}

The conjecture 2 implies that there is an infinite tower of light particles at infinite distance from any point inside the moduli space, where the effective field theory in the interior breaks down, and potentially a new description takes over. This is consistent with the following field theory fact.

Suppose there is a field $\varphi$, whose mass $m(\phi)$ is a function of the moduli field $\phi$. We assume that $m(\phi)$ is generically large in the moduli space $\mathcal{M}$ so that we can integrate out $\varphi$. Now suppose there is a particular point $\phi_{0} \in \mathcal{M}$ where $m\left(\phi_{0}\right)=0$. There the effective field theory with $\varphi$ integrated out breaks down. To see its effect on the moduli space metric, let us look at how the one-loop diagram of the $\varphi$ field modifies the kinetic term of $\phi$. If $\varphi$ is a scalar field, the coupling between $\varphi$ and $\phi$ is given by $\mathcal{L}_{i n t}=m(\phi)^{2} \varphi^{2}$, and it gives the one-loop correction:

$$
g_{i j}^{\text {one-loop }}=m(\phi)^{d-4} \frac{\partial m}{\partial \phi^{i}} \frac{\partial m}{\partial \phi^{j}} .
$$

Here $d$ is the spacetime dimensions of the Minkowski space where we are considering the gravity theory. Consider a point $\phi_{1}$ near $\phi_{0}$ where $m\left(\phi_{1}\right)=\epsilon \ll 1$ (in Planck units). The distance to $\phi_{1}$ measured by the quantum corrected metric in the above is given by

$$
d\left(\phi, \phi_{1}\right)=\int_{\epsilon} m^{\frac{d-4}{2}} d m=-\frac{2}{d-2} \epsilon^{\frac{d-2}{2}}+\text { finite. }
$$

Thus, a finite number of light particles does not generate infinite distance for $d>2$. We get the same result if the light particle is a fermion $\psi$ with an interaction $\mathcal{L}_{i n t}=m(\phi) \bar{\psi} \psi$.

On the other hand, a sum over one-loop effects of an infinite tower of light particles can produce a distance divergence in a quantum corrected metric. This field theory fact is consistent with our conjecture that an infinite distance singularity in the moduli space is correlated to an emergence of an infinite tower of light particles. Note, however, that we are not claiming that the infinite distance in moduli space is a direct consequence of having an infinite tower of massless states. Indeed it is possible to show that these are independent conjectures and one conjecture does not follow from the other. For example, 
the KK tower of light states (which can affect the distance in moduli space at one loop level) is not directly related to an infinite distance in the moduli space which is observable at tree level. Also sometimes there is an infinite tower of light states but at finite distance. This typically happens at conformal fixed points. For example consider M-theory compactified on a Calabi-Yau 3-fold near a point in moduli where a $\mathbf{P}^{2}$ inside the Calabi-Yau shrinks to a point. This leads to an infinite tower of light M2 branes wrapping shrinking cycles in $\mathbf{P}^{2}$. Nevertheless the distance in moduli space where $\mathbf{P}^{2}$ shrinks to zero size is finite.

\subsection{Observable Regions of Moduli Spaces}

For simplicity, let us consider the case when there is one massless scalar field $\phi$ and choose its parametrization so that the metric in the moduli space is flat: $d s^{2}=M_{P l}^{-2} d \phi^{2}$, where $M_{P l}$ is the Planck mass. This means that the value of $\phi$ is the geodesic length times $M_{P l}$.

Suppose $\phi$ is homogeneous in space. If it were not for gravity, $\phi$ would evolve linearly in time $t$. If we turn on the gravity and assume that the vacuum energy is dominated by the kinetic term of $\phi$, we have the Freedman-Robertson-Walker cosmology, where the Hubble friction slows down the scalar velocity $\dot{\phi}=\frac{d \phi}{d t}$ :

$$
\ddot{\phi}+d H \dot{\phi}=0, \quad H^{2}=\frac{\dot{\phi}^{2}}{M_{P l}^{2}},
$$

where $d$ is the spatial dimensions of flat directions. With the initial condition, $\dot{\phi}(t=0)=c$, we can integrate this to find

$$
\phi(t)=\phi(0)+\frac{M_{P l}}{d} \log \left(1+\frac{c}{M_{P l}} t\right) .
$$

In particular, $\phi(t)$ grows like log $t$ for large $t$.

If this description were valid for all $t$, the scalar field would continue to grow. However, the energy density of $\phi$ goes as $\dot{\phi}^{2} \sim 1 / t^{2} \rightarrow 0$. Eventually, other sources for $H$ become relevant. We should then write,

$$
H^{2}=\frac{\dot{\phi}^{2}}{M_{P l}^{2}}+H_{\text {others }}^{2} \sim\left(\frac{1}{d t}\right)^{-2}+H_{\text {others }}^{2},
$$

where $H_{\text {others }}$ represents contributions from other sources. When $t \gg 1 / H_{\text {others }}$, the velocity of the scalar field starts to decay exponentially, $\dot{\phi} \sim \exp \left(-d H_{\text {others }} t\right)$ and the evolution of $\phi$ is shut off. Thus we find that

$$
|\phi(t)-\phi(0)| \sim-\frac{M_{P l}}{d} \log H_{\text {others }}, \quad t \rightarrow \infty .
$$


If we identify $H_{\text {others }}$ with the mass scale $\epsilon$ for the low energy effective theory, this takes the same form as the logarithmic cutoff $T<-M_{P l} \log \epsilon$ for the geodesic length in the moduli space. This suggests that the classical evolution of the field $\phi$ cannot probe the region where the low energy effective theory breaks down by the appearance of an infinite tower of light particles. Similar ideas have been considered in [23,24].

It is clear from the above discussion that the logarithmic dependence of distance with the emergence of light mass scale is very natural from the field theory view point. It would be nice to try to more clearly relate the ideas from field theory with our conjectures about the appearance of light modes at infinite corners of moduli space.

\section{Concluding Thoughts}

We have raised a number of conjectures about the geometry of scalar moduli in a consistent quantum theory of gravity. We find determination of whether or not these and similar conjectures are true or false as critical to a deeper understanding of what constitutes the universality class of quantum gravitational theories. Ultimately we would need to learn more: Even if we establish they are correct we would need to know why! This would presumably involve deep questions of gravity, such as questions involved in the context of black hole. It would be very satisfying if we can relate such geometric questions on moduli of scalar fields to deeper issues involving black holes and holography. We hope raising such question will contribute to asking deeper questions in the context of quantum gravity and how an effective field theory can be possibly consistent with it.

\section{Acknowledgments}

We would like to thank N. Arkani-Hamed for collaboration at an early stage of this work. In addition we would like to thank D. Calegari, M. Douglas, S. Kachru, Z. Lu, D. Morrison, L. Motl, A. Neitzke, H. Oh, M. Roček, S. Shenker, A. Strominger, A. Van Proeyen, and S.-T. Yau for valuable discussions. H.O. is grateful to the theory group of Harvard University for their hospitality. H.O. also thanks the organizers of the conference to celebrate the 60th birthday of Michael Green, where a preliminary version of this work was presented.

The research of H.O. is supported in part by DOE grant DE-FG03-92-ER40701. The research of C.V. is supported in part by NSF grants PHY-0244821 and DMS-0244464. 


\section{References}

[1] C. Vafa, "The string landscape and the swampland," hep-th/0509212.

[2] M. R. Douglas and Z. Lu, "Finiteness of volume of moduli spaces," hep-th/0509224.

[3] N. Arkani-Hamed, L. Motl, A. Nicolis and C. Vafa, "The string landscape, black holes and gravity as the weakest force," hep-th/0601001.

[4] M. R. Douglas, talk at the Strings 2005 Conference, http://www.fields.utoronto.ca/ audio/05-06/strings/douglas/.

[5] S. Kachru, J. McGreevy and P. Svrcek, "Bounds on masses of bulk fields in string compactifications," hep-th/0601111.

[6] D. V. Anosov, "Geodesic flows on closed Romanian manifolds with negative curvature," Proc. Steklov Inst. Mathematics 90, 209 (1967).

[7] E. Hopf, Leipzig Ber. Verhandl. Sächs. Akad. Wiss. 91, 261 (1939); For discussion of the ergodicity of geodesic flows in the context of string compactification, see G. W. Moore, "Finite in all directions," hep-th/9305139.

[8] C. C. Moore, "Ergodicity of flows on homogeneous spaces," Amer. J. Math., 88 (1966) 154.

[9] C. Vafa, "Quantum symmetries of string vacua," Mod. Phys. Lett. A 4, 1615 (1989).

[10] P. S. Aspinwall and D. R. Morrison, "String theory on K3 surfaces," hep-th/9404151.

[11] P. Candelas, X. C. De La Ossa, P. S. Green and L. Parkes, "A pair of Calabi-Yau manifolds as an exactly soluble superconformal theory," Nucl. Phys. B 359, 21 (1991).

[12] Z. Lu and X. Sun, "On the Weil-Petersson volume and the first Chern class of the moduli space of Calabi-Yau manifolds," Commun. Math. Phys. 261, 297 (2006); math.dg/0510021.

[13] E. A. Rodland, "The Pfaffian Calabi-Yau, its mirror, and their link to the Grassmannian $G(2,7)$," Compositio Math. 122, 135 (2000).

[14] J. A. Wolf, "Complex homogeneous contact manifolds and quarternionic symmetric spaces," J. Math. Mech. 14, 1033 (1965).

[15] J. Bagger and E. Witten, "Matter couplings in $\mathcal{N}=2$ supergravity," Nucl. Phys. B 222, 1 (1983).

[16] O. DeWolfe, A. Giryavets, S. Kachru and W. Taylor, "Type IIA moduli stabilization," JHEP 0507, 066 (2005); hep-th/0505160.

[17] B. de Wit and A. Van Proeyen, "Potentials and symmetries of general gauged $\mathcal{N}=2$ supergravity - Yang-Mills models," Nucl. Phys. B 245, 89 (1984).

[18] M. R. Douglas, H. Ooguri and S. H. Shenker, "Issues in (M)atrix model compactification," Phys. Lett. B 402, 36 (1997); hep-th/9702203.

[19] M. R. Douglas and H. Ooguri, "Why matrix theory is hard," Phys. Lett. B 425, 71 (1998); hep-th/9710178. 
[20] M. R. Douglas and G. W. Moore, "D-branes, quivers, and ALE instantons," hepth/9603167.

[21] M. R. Douglas and B. R. Greene, "Metrics on D-brane orbifolds," Adv. Theor. Math. Phys. 1, 184 (1998); hep-th/9707214.

[22] S. Katz, A. Klemm and C. Vafa, "M-theory, topological strings and spinning black holes," Adv. Theor. Math. Phys. 3, 1445 (1999); hep-th/9910181.

[23] T. Banks, "A critique of pure string theory: Heterodox opinions of diverse dimensions," hep-th/0306074.

[24] T. Banks, M. Dine and E. Gorbatov, "Is there a string theory landscape?," JHEP 0408, 058 (2004); hep-th/0309170. 